\title{
Aplicação de tecnologias para melhoramento das características funcionais da clara de ovo desidratada: Uma revisão
}

\author{
Application of technologies to improve functional characteristics of the egg white powder: A review \\ Aplicación de tecnologías para mejorar las características funcionales de la clara de huevo \\ deshidratada: Una revisión
}

Recebido: 03/06/2021 | Revisado: 09/06/2021 | Aceito: 10/06/2021 | Publicado: 26/06/2021

Jéssica Mayuri Oma

ORCID: https://orcid.org/0000-0003-1439-6774 Universidade Estadual de Maringá, Brasil

E-mail: mayurii.omae@gmail.com

Suelen Siqueira dos Santos

ORCID: https://orcid.org/0000-0003-4484-7425 Universidade Federal de Uberlândia, Brasil E-mail: suelensiqueira.eng@gmail.com

Grasiele Scaramal Madrona

ORCID: https://orcid.org/0000-0002-8837-8424 Universidade Estadual de Maringá, Brasil E-mail: gsmadrona@uem.br

\begin{abstract}
Resumo
A clara de ovo desidratada é um produto bastante utilizado em diversas aplicações, principalmente na confeitaria e panificação, entretanto, ao passar por processos industriais acaba perdendo algumas de suas características e funcionalidades. Para que isso não aconteça, pesquisas vêm sendo realizadas em busca de métodos de tratamento térmico a seco que possam melhorar suas características, e oferecer ainda maior segurança microbiológica. Assim, o objetivo desse trabalho foi realizar uma revisão bibliográfica das pesquisas mais recentes nesse âmbito, utilizando as bases de dados Pubmed, Scientific Electronic Library Online (Scielo) e o portal de periódicos da CAPES, tendo como descritores "clara de ovo desidratada" e "egg white powder". A pesquisa retornou com um total geral de 4684 artigos sendo que deles, 12 (da plataforma CAPES) eram referentes à aplicação de tratamento térmico a seco. Os resultados mostraram que a aplicação dessas tecnologias tem obtido êxito, porém, apenas em escala laboratorial, não sendo ainda avaliada a viabilidade econômica para aplicação industrial.
\end{abstract}

Palavras-chave: Clara de ovo desidratada; Tratamento térmico a seco.

\begin{abstract}
Dehydrated egg white is a product widely used in several applications, mainly in confectionery and bakery, however, when going through industrial processes it ends up losing some of its characteristics and functionalities. For that not to happen, research has been carried out looking for methods of dry thermal treatment that improve its characteristics and may also offer even greater microbiological safety. Thus, the objective of this work was to carry out a bibliographic review of the most recent research in this area, using Pubmed, Scientific Electronic Library Online (Scielo), and the CAPES periodical portal as databases and having as descriptors "clara de ovo desidratada" and "egg white powder". The research returned with a grand total of 4684 articles, of which 12 (from the CAPES platform) were related to the application of dry heat treatment. The results showed that the application of these technologies has been successful, however, only on a laboratory scale, and the economic viability for industrial application has not yet been evaluated.
\end{abstract}

Keywords: Egg white powder; Dry thermal treatment.

\section{Resumen}

La clara de huevo deshidratada es un producto muy utilizado en varias aplicaciones, principalmente en repostería y panadería, sin embargo, al pasar por procesos industriales acaba perdiendo algunas de sus características y funcionalidades. Para evitar que esto suceda, se han realizado investigaciones en busca de métodos de tratamiento con calor seco que puedan mejorar sus características y ofrecer una seguridad microbiológica aún mayor. Así, el objetivo de este trabajo fue realizar una revisión bibliográfica de las investigaciones más recientes en esta área, utilizando las bases de datos Pubmed, Scientific Electronic Library Online (Scielo) y el portal de revistas CAPES, con los descriptores "clara de ovo desidratada" y "egg white powder". La búsqueda arrojó un total de 4684 artículos, 12 de los cuales (de la plataforma CAPES) estaban relacionados con la aplicación de tratamiento de calor seco. Los resultados mostraron que 
la aplicación de estas tecnologías ha tenido éxito, sin embargo, solo a escala de laboratorio, y aún no se ha evaluado la viabilidad económica para la aplicación industrial.

Palabras clave: Clara de huevo en polvo; Tratamiento con calor seco.

\section{Introdução}

A clara de ovo é um produto versátil e rico em nutrientes podendo ser aplicada em bolos, doces, merengues, embutidos e outros onde confere aeração, capacidade de retenção de água, força de gel e outras funcionalidades, porém, apresenta baixo shelf life quando in natura ( $\mathrm{Li}$ et al., 2018). Sendo assim, uma forma que a indústria investiu para aproveitar melhor seus benefícios, foi sua produção na versão desidratada, onde o produto apresenta diversas de suas funcionalidades e ainda maior facilidade de armazenamento, maior segurança microbiológica e shelf life estendido (Ma et al., 2019).

De acordo com o tipo de aplicação proposto a clara de ovo pode não ser tão interessante na forma desidratada, uma vez que a alta temperatura desnatura parte das proteínas prejudicando seu desempenho. Sendo assim, vem-se estudando mecanismos de melhorar características físico-químicas e propriedades funcionais da clara de ovo desidratada viáveis à indústria (Wang et al., 2020).

Um dos mecanismos utilizados é o tratamento térmico a seco do produto já desidratado. Esse tratamento já é de conhecimento da indústria, sendo hoje o "hot room" um método tradicional utilizado como pasteurização, não apenas visando a garantia microbiológica do produto, mas também usado para proporcionar melhoria em suas funcionalidades (Wei et al., 2020).

Apesar do "hot room" apresentar bons resultados e atingir seu objetivo, é um método lento e caro (demanda muita energia), o que tem levado a pesquisas de formas alternativas de tratamento térmico a seco com menor tempo de aplicação e que apresente os mesmos resultados em termos de funcionalidade da clara de ovo desidratada (Boreddy et al., 2016a).

Alguns dos tratamentos térmicos que vem sendo estudados são tratamentos de radiofrequência e micro-ondas, conhecidos como métodos de aquecimento dielétrico. Eles aquecem o alimento pela conversão da energia elétrica em energia térmica e, dessa forma, podem atingir a temperatura no centro do produto de forma mais rápida e, assim, reduzir o tempo de tratamento térmico tradicional (Boreddy et al., 2016b).

Não somente os tratamentos térmicos a seco, mas também se tem estudado as interações que acontecem com as proteínas da clara de ovo. Sabe-se que as propriedades das proteínas são modificadas e, assim, busca-se entender de que forma essas modificações afetam as propriedades funcionais da clara como um todo (Lechevalier et al., 2017).

Há também outros estudos que visam à melhora da funcionalidade, propriedades da clara em conjunto, melhoria de algum aspecto específico e também tecnologias que garantam a segurança microbiológica da clara de ovo desidratada. Dentre eles pode-se citar a utilização de gomas, fermentação, carbonatação, ultrassom e ainda, ajustes em parâmetros de tecnologias já utilizadas. Assim, o objetivo desse estudo, foi realizar uma revisão bibliográfica sistematizada de pesquisas recentes nesse âmbito, utilizando como base de dados a Pubmed, Scientific Electronic Library Online (Scielo) e o portal de periódicos da CAPES.

\section{Metodologia}

Esta revisão bibliográfica é de natureza qualitativa, fundamentada em um levantamento de dados bibliográficos em bases de dados Pubmed, Scientific Electronic Library Online (Scielo) e o portal de periódicos da CAPES, com os seguintes descritores: "clara de ovo desidratada" e "egg white powder", sendo considerados os presentes no título e/ou resumo. Devido à grande quantidade de artigos que resultaram da busca na base CAPES, aplicou-se nesse caso o uso de aspas no descritor.

Para otimizar a qualidade da pesquisa foram aplicados filtros na busca, do ano de 2016 a 2020, e separados apenas artigos. Como método de exclusão foram desconsiderados artigos em que o foco não eram as características físico-químicas e/ou 
propriedades funcionais da clara de ovo desidratada e claras de ovos obtidas somente de galinhas. Também não foram considerados monografias e artigos de revisão. Essas estratégias utilizadas foram baseadas em protocolos específicos de busca sugeridos por Galvão e Ricarte (2019).

\section{Resultados e Discussão}

A pesquisa realizada com os descritores clara de ovo desidratada e egg white powder resultou em um grande número de artigos somente para o descritor em inglês, sendo necessários utilizar aspas no descritor "egg white powder" para reduzir a quantidade de resultados relevantes. A tabela 1 mostra a quantidade de artigos encontrados nas bases de dados utilizadas e de acordo com o descritor.

Tabela 1. Quantidade de artigos resultantes da pesquisa realizada nas bases de dados Scielo, Pubmed e CAPES para os descritores Clara de Ovo Desidratada e Egg White Powder (com e sem aspas).

\begin{tabular}{lccc}
\hline & Resultado de Busca de artigos no período de 2016 - 2020 & \\
\hline Descritor/Base de dados & SCIELO & PUBMED & CAPES \\
\hline Clara de Ovo Desidratada & 0 & 0 & 0 \\
Egg White Powder & 3 & 62 & 4.619 \\
"Egg White Powder" & 0 & 6 & 174 \\
\hline
\end{tabular}

Fonte: Autores.

Ao pesquisar por egg white powder, apesar do grande número de artigos encontrados, a maioria estudava a clara como ingrediente, onde o foco não eram suas características funcionais em si, e sim o seu efeito aplicado em outros alimentos e produtos. Da pesquisa na plataforma Scielo, os 3 artigos encontrados apresentaram esse foco, e dessa forma nenhum foi utilizado. Já a pesquisa realizada na plataforma Pubmed, apenas dois apresentaram as aplicações desejadas, porém, os mesmos também foram encontrados da plataforma CAPES. Sendo assim, pode-se considerar que os artigos utilizados nessa revisão foram todos extraídos desta plataforma.

Observou-se que, dentre os artigos selecionados, praticamente metade deles são de pesquisadores chineses, seguido pelos dos Estados Unidos e depois dividido entre outros países conforme ilustra a Figura 1. 
Figura 1. Quantidade de artigos por país de origem.

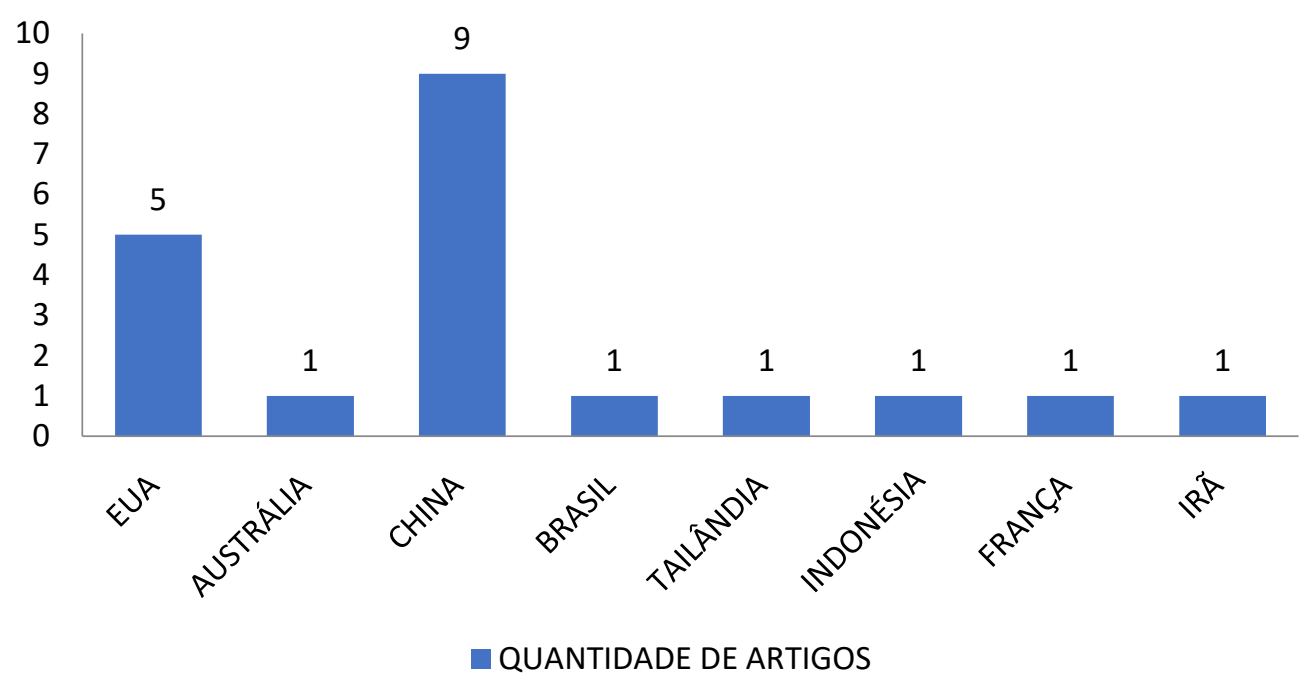

Fonte: Autores.

A quantidade de artigos por país mostra que no tema em questão os países China e Estados Unidos são os que mais apresentam pesquisas recentes. Esse fato pode ser explicado por serem os países que mais produzem e consomem ovos no mundo, como descrito por Amaral et al. (2016).

Os artigos selecionados foram avaliados individualmente sendo que 4 tratam do efeito do tratamento térmico a seco em geral nas características funcionais da clara desidratada, 4 da aplicação de micro-ondas, 3 da aplicação de radiofrequência e os 9 outros se dividem entre aplicações de outros métodos e utilização de ingredientes visando melhorar as características funcionais da clara de ovo desidratada.

A Tabela 2 apresenta os principais temas abordados e a quantidade de artigos que discorrem sobre eles. 
Tabela 2. Quantidade de artigos por tema.

\begin{tabular}{|c|c|c|}
\hline Quantidade de artigos & Tema & Referências \\
\hline 4 & $\begin{array}{l}\text { Efeito de tratamento térmico a seco em } \\
\text { clara de ovo em desidratada }\end{array}$ & $\begin{array}{l}\text { Chang et al. (2020), Lechevalier et al. } \\
\text { (2017), Ma et al. (2019), Ma et al. } \\
\text { (2020) }\end{array}$ \\
\hline 4 & $\begin{array}{l}\text { Efeito da aplicação de micro-ondas em } \\
\text { clara de ovo desidratada }\end{array}$ & $\begin{array}{l}\text { Li et al. (2018), Li et al. (2019), Li et al. } \\
\text { (2020) e Wang et al. (2020) }\end{array}$ \\
\hline 3 & $\begin{array}{l}\text { Efeito da aplicação de radiofrequência } \\
\text { em clara de ovo desidratada }\end{array}$ & $\begin{array}{l}\text { Kar et al. (2020), Boreddy et al. } \\
\text { (2016a), Wei et al. (2020) }\end{array}$ \\
\hline 1 & $\begin{array}{l}\text { Efeito da aplicação de ultrassom em } \\
\text { clara de ovo desidratada }\end{array}$ & Stefanović et al. (2017) \\
\hline 1 & $\begin{array}{c}\text { Método matemático para calcular a } \\
\text { drenagem da clara em neve }\end{array}$ & Faezian et al. (2019) \\
\hline 1 & $\begin{array}{c}\text { Aplicação e efeito da fermentação na } \\
\text { clara de ovo }\end{array}$ & Pratama et al. (2019) \\
\hline 1 & Efeito da carbonatação & Katekhong et al. (2018) \\
\hline 1 & $\begin{array}{l}\text { Efeito da gelatina de escama de peixe na } \\
\text { clara em neve }\end{array}$ & Huanga et al. (2017) \\
\hline 1 & $\begin{array}{l}\text { Comparação entre os métodos de } \\
\text { radiofrequência e micro-ondas e relação } \\
\text { entre temperatura e umidade na clara de } \\
\text { ovo desidratada }\end{array}$ & Boreddy et al. (2016b) \\
\hline 1 & $\begin{array}{l}\text { Variação de temperatura de secagem } \\
\text { por spray drying e efeito na clara de ovo } \\
\text { desidratada }\end{array}$ & Wattinee \& Sanguansri (2018) \\
\hline 1 & $\begin{array}{c}\text { Método para quantificação de } \\
\text { halogênios e enxofre em ovo pó e } \\
\text { derivados }\end{array}$ & Mesko et al. (2020) \\
\hline 1 & $\begin{array}{l}\text { Efeito de sistemas geradores de radicais } \\
\text { hidroxila na clara de ovo desidratada }\end{array}$ & Wang et al. (2018) \\
\hline
\end{tabular}

Fonte: Autores.

De acordo com os dados demonstrados na Tabela 2, nota-se que 12 dos 20 artigos tratam de aplicações de métodos térmicos a seco em clara de ovo desidratada e deles todos apresentaram resultados satisfatórios na melhoria das propriedades de gel, formação de espuma e solubilidade. Já com os outros 8 artigos foi possível identificar a importância das propriedades da clara de ovo desidratada e métodos para mensurar essas propriedades.

As pesquisas que utilizaram o método de radiofrequência (Kar et al., 2020; Boreddy et al., 2016a) apresentaram resultados satisfatórios para a melhora das propriedades físico-químicas e funcionalidade além de ser um método mais rápido e mais acessível comparado ao método de "hot room". 
Wei et al. (2020) validaram o processo de radiofrequência como uma alternativa para tratamento térmico mostrando ser um método eficaz para eliminação de salmonela, enquanto Boreddy et al. (2016a) identificaram um melhor desempenho ao aplicar o produto para obtenção de clara em neve e, assim como Kar et al. (2020) destacaram melhoria na propriedade de força de gel.

A aplicação da radiofrequência para a obtenção de uma melhor clara em neve é uma vantagem devido ao menor tempo de tratamento quando comparado com o método tradicional ("hot room"), onde seriam necessários de 10 a 14 dias, e com a radiofrequência, em $24 \mathrm{~h}$ se atinge o mesmo resultado.

Ainda na propriedade de força de gel, Ma et al. (2020) também obtiveram melhora considerável na força de gel da clara de ovo desidratada ao realizar pré-tratamento com ressonância magnética nuclear de baixo campo.

Já nos artigos que avaliaram a aplicação de micro-ondas, os autores Li et al. (2018), Li et al. (2019), Li et al. (2020) e Wang et al. (2020) apresentaram resultados muito semelhantes tendo todos em comum resultado satisfatório na melhora da formação e estabilidade da clara em neve, o que ocorreu na aplicação de maior potência de micro-ondas, sendo que Wang et al. (2020) além disso, constataram que o tratamento com micro-ondas poderia restaurar a estrutura secundária da proteína da clara do ovo desidratada para a mesma proteína natural do ovo líquido (desidratada), o que tornaria o produto ainda mais vantajoso.

Também foi verificado pelos três autores que a aplicação de micro-ondas pode ocasionar uma melhoria na solubilidade dependendo da potência utilizada e se em pH alcalino. Sendo que a solubilidade está diretamente relacionada à propriedades de emulsão, ou seja, quanto maior a solubilidade, melhor será a formação de clara em neve.

Em todos os estudos abordados foi observado que os tratamentos térmicos a seco influenciam diretamente na estrutura das proteínas que compõem a clara de ovo, de forma que de acordo com o tempo de exposição e intensidades aplicadas das tecnologias utilizadas pode-se obter resultados diferentes e em propriedades diferentes de acordo com o objetivo pretendido.

\section{Conclusão}

A quantidade de artigos encontrados sobre a utilização de clara de ovo desidratada e métodos para melhorar suas características físico-químicas e funcionais demonstram a relevância do assunto, embora ainda não muito explorado.

Os resultados apresentados nessa revisão bibliográfica mostram que as pesquisas no campo de tecnologias para aplicação de tratamentos térmicos a seco em clara de ovo desidratada têm aumentado e mostrado resultados satisfatórios, porém, em escala laboratorial. Apesar dos bons resultados e aplicação à indústria, não foi levantado em nenhum deles a viabilidade econômica para aplicação em escala industrial, nem avaliação sensorial em aplicação em produto, o que seria interessante para trabalhos futuros. Além disso, também sugere-se realizar um levantamento sobre a avaliação da vida de prateleira da clara de ovo desidratada, bem como a adição de outros ingredientes alimentares, como edulcorantes, corantes, saborizantes, conservantes, entre outros.

\section{Referências}

Amaral, G. F., Guimarães, D., Nascimento, J. C. \& Custodio, S. (2016). Avicultura de postura: estrutura da cadeia produtiva, panorama do setor no Brasil e no mundo e o apoio do BNDES. BNDES Setorial, 43, 167-207.

Boreddy, S. R., Thippareddi, H., Froning, G. \& Subbiah, J. (2016a). Novel Radiofrequency-Assisted Thermal Processing Improves the Gelling Propertiesof Standard Egg White Powder. Journal of Food Engineering, 168, 60-67.

Boreddy, S. R. \& Subbiah, J. (2016b). Temperature and moisture dependent dielectric properties of egg white powder. Journal of Food Science, 81 , 665-671.

Chang, C., Xu, Y., Shi, M., Su, Y., Li, X., Li, J. \& Yang, Y. (2020). Effect of dry-heat and guar gum on properties of egg white powder: Analysis of forming capacity and baking performance. Food Hydrocolloids, 99, 1-6. 
Faezian, A., Yeganehzad, S. \& Tighchi, H. A. (2019). A simplified model to describe drainage of egg white powder foam containing additives. Chemical Engineering Science, 195, 631-641.

Galvão, M. C. B., \& Ricarte, I. L. M. (2019). Systematic literature review: concept, production and publication. Logeion: Filosofia da Informação, 6, 57-73.

Huanga,T., Tu, Z., Wanga, H., Shangguan, X., Zhang, L., Niua, P. \& Sha, X. (2017). Promotion of foam properties of egg white protein by subcritical water pre-treatment and fish scales gelatin. Colloids and Surfaces A: Physicochem. Eng. Aspects, 512, 171-177.

Kar, A., Wei, X., Majumder, K., Eskridge, K., Handa, A. \& Subbiah, J. (2020). Effect of traditional and radiofrequency assisted thermal processing on the gel firmness of egg white powder. LWT Food Science and Technology, 133, 1-6.

Katekhong, W., Bhandari, B.; Jittanit, W., Charoenrein, S. (2018). Effect of carbonation of fresh egg white prior to spray drying on physical and functional properties of powder. Drying Technology, 36, 1224-1235.

Lechevalier, V., Guerin-Dubiard, C., Ant, M., Beaumal, V., Briand, E. D., Gillard, A., Le Gouar, Y., Musikaphun, N., Pasco, M., Dupont, D. \& Nau, F. (2017). Effect of dry heat treatment of egg white powder on its functional, nutritional and allergenic properties. Journal of Food Engeneering, $195,40-51$.

Li, P., Sun, Z., Ma, M., Jin, Y. \& Sheng, L. (2018). Effect of microwave-assisted phosphorylation modification on the structural and foaming properties of egg white powder. LWT Food Science and Technology, 97, 151-156.

Li, P., Jin, Y. \& Sheng, L. (2020). Impact of microwave assisted phosphorylation on the physicochemistry and rehydration behaviour of egg white powder. Food Hydrocolloids, 100, 1-6.

Li, P., Sheng, L. \& Jin, Y. (2019). Using microwave-assisted phosphorylation to improve foaming and solubility of egg white by response surface methodology. Poultry Science, 98, 7110-7117.

Ma, Y., Zhaob, Y. \& Chi, Y. (2019). Changes in the gel characteristics of two hen egg white powders modified by dry heating and the Maillard reaction during long-term storage. LWT Food Science and Technology, 109, 123-129.

Ma, Y., Shan, A., Wang, R., Zhao, Y. \& Chi, Y. (2020). Characterization of egg white powder gel structure and its relationship with gel properties influenced by pretreatment with dry heat. Food Hydrocolloids, 110, 1-9.

Mesko, M. F., Toralles, I. G., Junior, G. S. C., Rondan, F. S., Costa, V. C., Hartwig, C. A. \& Scaglioni, P. T. (2020). Ion chromatography coupled to mass spectrometry as a powerful technique for halogens and sulfur determination in egg powder and its fractions. Wiley Rapid Communications in Mass Spectrometry, $34,1-9$.

Pratama, A., Putranto, W. S., Suradi, K., Adawiyah, R., Risanawati. \& Ninda, C. (2019). The effect of Kluyveromyces lactis starter concentration and fermentation time to the physicochemical and functional properties of egg white powder. Jurnal Ilmu dan Teknologi Hasil Ternak, 14, 117-125.

Stefanović, A. B., Jovanović, J. R., Dojčinović, M. B., Lević, S. M., Nedović, V. A., Bugarski, B. M. \& Knežević-Jugović, Z. D. (2017). Effect of the Controlled High-Intensity Ultrasound on Improving Functionality and Structural Changes of Egg White Proteins. Food Bioprocess Techol, 10, $1224-1239$.

Wang, J., Zhao, Y., Niu, S., Wang, X. \& Chen, F. (2018). Effect of oxidation induced by hydroxyl radical-mediated model on molecular structural and physical character of egg white powder. International Journal of Food Science and Techology, 53, 2281-2289.

Wang, X., Gu, L., Su, W., Li, J., Yang, Y. \& Chang, C. (2020). Microwave technology as a new strategy to induce structural transition and foaming properties improvement of egg white powder. Food Hydrocolloids, 101, 1-6.

Wattinee, K.\& Sanguansri, C. (2018). Influence of spray drying temperatures and storage conditions on physical and functional properties of dried egg white. Drying Technology, 36, 169-177.

Wei, X., Lau, S. K., Reddy, B. S. \& Subbiah, J. (2020). A microbial challenge study for validating continuous radio-frequency assisted thermal processing pasteurization of egg white powder. Food Microbiology, 85, 1-7. 